\title{
The Mutual Influence of Gaze and Head Orientation in the Analysis of Social Attention Direction
}

\author{
Stephen R. H. Langton \\ Department of Psychology, University of Stirling
}

Running Head: SOCIAL ATTENTION

Correspondence to: Dr. S. R. H. Langton, Dept. of Psychology, University of Stirling, Stirling, FK9 4LA, Scotland.

E-Mail: srhl1@stirling.ac.uk

Fax: +44 (0) 1786-467-641 


\begin{abstract}
Three experiments are reported that investigate the hypothesis that head orientation and gaze direction interact in the processing of another individual's direction of social attention. A Strooptype interference paradigm was adopted in which gaze and head cues were placed into conflict. In separate blocks of trials, participants were asked to make speeded keypress responses contingent on either the direction of gaze, or the orientation of the head displayed in a digitised photograph of a male face. In Experiments 1 and 2 head and gaze cues showed symmetrical interference effects. Compared with congruent arrangements, incongruent head cues slowed responses to gaze cues, and incongruent gaze cues slowed responses to head cues, suggesting that head and gaze are mutually influential in the analysis of social attention direction. This mutuality was also evident in a cross-modal version of the task (Experiment 3) where participants responded to spoken directional words whilst ignoring the head/gaze images. It is argued that these interference effects arise from the independent influences of gaze and head orientation on decisions concerning social attention direction.
\end{abstract}


The ability to analyse where another individual is directing their attention has important behavioural and communicative consequences for the observer. For example, humans and many other species tend to direct their gaze toward objects or regions of space that are of immediate interest to them. A gaze in your direction, therefore, usually signals that the owner of these eyes has a particular interest in you, which may be a good thing - you are a potential mate, or a bad thing - you are a potential lunch. Most mammals and non-human primates wisely default to assuming something like the latter, treating mutual gaze as a signal of threat or dominance, and consequently responding with their own threat display, or with fear and avoidance behaviours (e.g., Argyle \& Cook, 1976; Mendelson, Haith, \& Goldman-Rakic, 1982). Although humans react in similar ways to prolonged gaze from strangers (e.g., Argyle \& Cook, 1976), we also use and interpret direct gaze as an expression of certain interpersonal attitudes such as love or attraction, and as a cue for signalling turn taking in conversation (Kendon, 1967).

Whilst there are clear advantages in the rapid and efficient detection of direct gaze from another individual (e.g., von Grünau \& Anston, 1995), it is equally important to be able to compute the direction of another's attention even when it is oriented elsewhere in the environment. A sudden change in another's gaze away from an observer, for instance, will often signal a significant environmental event such as the approach of a predator, a meal or an attractive conspecific (see Byrne \& Whiten, 1991). The ability to compute their gaze direction, and then to orient one's own attention in this direction will therefore facilitate the detection, and thence the response to these events (Driver, Davis, Kidd, Maxwell, Ricciardelli \& Baron-Cohen, in press; Langton \& Bruce, in press). The detection and response to another's gaze is also important in communicative contexts where gaze might be used as a deictic signal, indicating the referent of a remark, or the current focus of interest. Indeed, the deictic function of gaze may well play a role in vocabulary acquisition by toddlers as by following a speaker's line of regard, the infant can determine the intended referent of an unfamiliar word (Baldwin, 1991).

Although the eyes provide a reliable cue to where another individual is currently directing their attention, this information could also be gleaned from other social signals such as the 
orientation of the head, the angle of body posture or the direction of a pointing gesture. Indeed, in the absence of information from the eyes, these other cues may be the only reliable indicators of the focus of another's attention. The aim of this paper is to explore the suggestion that information derived from the orientation of the head is routinely processed, and along with gaze information, contributes to the computation of another's direction of attention.

\section{Social Attention Detection}

Given the amount of information carried by the eyes, and the rather poor outlook for the individual who is unable to determine rapidly and efficiently where another animal is looking, it is perhaps not that surprising that we seem to have evolved a functionally separate mechanism devoted to the task of gaze perception. The evidence for this proposal originates from both neuropsychological and neurophysiological studies. The former have revealed a double dissociation between face recognition and gaze perception abilities, suggesting that these two aspects of face processing proceed independently in the information processing system. Campbell, Heywood, Cowey, Regard \& Landis (1990) describe a patient who was relatively unimpaired at gaze processing tests but was severely impaired on tests of facial recognition. Evidence of the reverse dissociation comes from Perrett, Hietanen, Oram, \& Benson (1992) who note a patient who was markedly impaired on gaze recognition tasks but only mildly impaired in the recognition of famous faces.

Neurophysiological work suggests that gaze detection is one of the functions of the superior temporal sulcus (STS) region of the primate temporal lobe. Single cell recording studies performed by Perrett and his colleagues have identified cells in this region of the macaque brain which respond selectively to the direction of gaze (e.g., Perrett, Smith, Potter, Mistlin, Head, Milner, \& Jeeves, 1985; Perrett, Hietanen, Oram, \& Benson, 1992). Furthermore, Heywood and Cowey (1992) showed that the removal of this region of the macaque cortex impaired gaze direction judgements by these monkeys but did not affect their performance on other face processing tasks. Together, these findings seem to indicate the existence of a functionally separate gaze detection module. Indeed, based largely on this kind of evidence, Baron-Cohen 
$(1994,1995 a, 1995 b)$ has proposed the existence of just such a "neurocognitive mechanism". His Eye Direction Detector (EDD) functions to detect the presence of eyes, or eye-like stimuli, and computes whether the eyes are directed toward it or toward another object in the environment. For Baron-Cohen, the ability to detect eye gaze direction, and then to use this information to establish joint attention, form important precursors in the development of a child's understanding of mental state concepts such as desire, intention, pretence and belief.

Whilst the eyes are of undoubted importance in the determination of where another is directing their attention, other cues such as head orientation, body posture or even pointing gestures may also provide important information in this regard. Indeed, there is some evidence that the mechanisms responsible for detecting gaze direction (i.e., the EDD) interact with mechanisms that process other information from the face and body. The work of Perrett and his colleagues has indicated that individual cells in the STS region of the macaque brain respond to conjunctions of eye, head and body position (e.g., Perrett et al., 1992). Thus, cells which respond preferentially to eyes directed downwards also respond when the head is oriented downwards and when the body adopts a quadrupedal posture. On the basis of these findings, Perrett and his colleagues argue that the STS cells play a role in analysing "social attention". The idea is that an STS cell receives inputs from cells selective for the visual appearance of particular head, gaze and posture orientations. Thus, in addition to a simple Eye Direction Detector, Perrett and Emery (1994) postulate the existence of a more general "Direction of Attention Detector" which combines information from these separate social signals in order to determine the direction of another's social attention.

Perrett et al. (1992) offer a hypothesis regarding how information from eyes, head and body might be combined in this direction of attention detector. Information from the eyes can directly inhibit cells coding an inappropriate head direction but not vice-versa. Similarly, information specifying a particular head angle can inhibit cells coding an inappropriate postural orientation but not vice-versa. This combination of connections ensures that information from gaze cues overrides that from head cues, and in turn, information from head cues overrides that derived 
from body cues. Attention direction can therefore be computed under a variety of viewing conditions. If the eyes are in view, input to the STS cells from head direction and body posture is inhibited so that attention direction is signalled by gaze cues. However, if the face is viewed at a distance or the eyes are obscured by shadow, the system defaults to signalling the direction of attention from the orientation of the head, or if this too is obscured, from the orientation of the body.

However, a relevant question is whether or not the neurophysiological observations correlate with observable behaviour. Do neurologically intact human participants behave in a fashion consistent with the idea that head and gaze cues interact in the processing of social attention direction, and is this interaction based on a hierarchy of cues as Perrett et al suggest? In fact, a number of behavioural studies have hinted that information extracted from the head plays a greater role in the computation of social attention direction than Perrett et al's model might suggest. Vecera and Johnson (1995) showed that participants' sensitivity to gaze direction in schematic faces was poorer when the eyes were in the context of an inverted or scrambled face compared to an upright face. More recently, Jenkins (1998) obtained a similar result using photographs of real faces and, in addition, demonstrated that sensitivity to gaze direction was compromised even when the eyes remained upright in the context of an inverted face. These findings have provided convincing evidence that the context provided by the face or head can influence the processing of gaze direction. However, some earlier studies have suggested that, more specifically, the context provided by the orientation of the head is influential in the perception of gaze direction. 


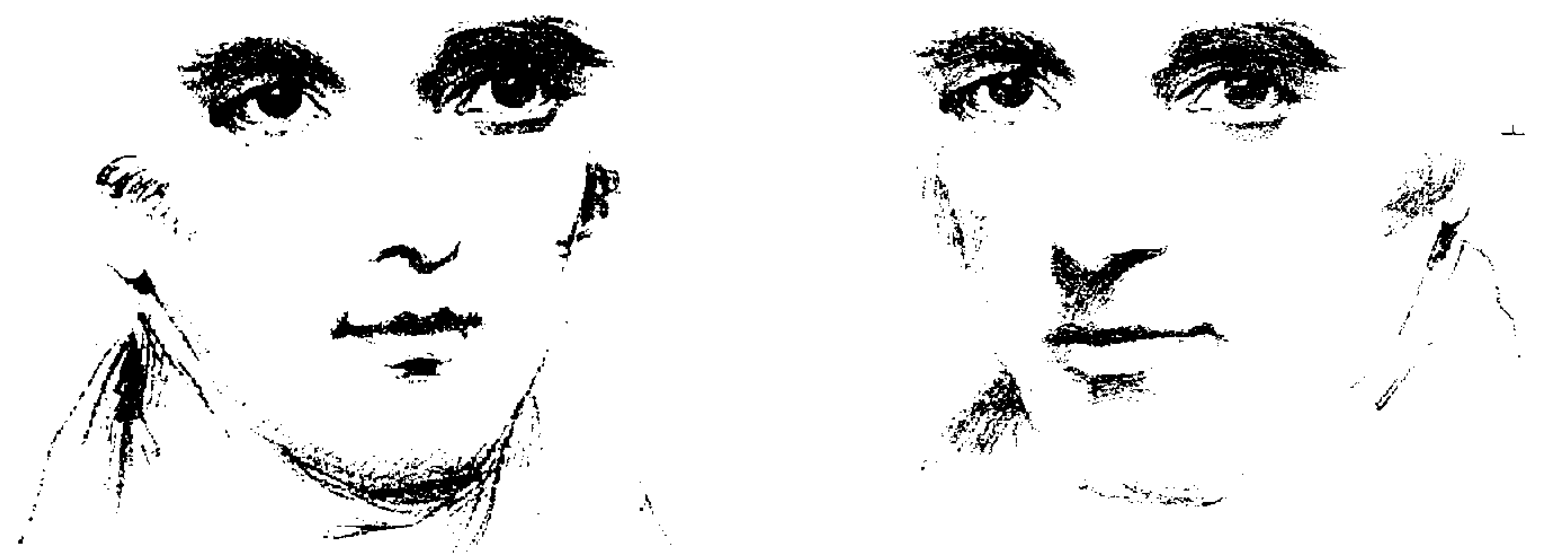

Figure 1. Examples of Wollaston's (1824) demonstrations that head orientation influences the perceived direction of gaze.

In the early part of the nineteenth century, William Wollaston (1824, cited in Bruce \& Young, 1998) noted that our judgements of eye gaze direction are not based solely on the position of the iris and pupil relative to the whites of the eyes. The illustrations taken from his paper (see Figure 1) clearly demonstrate how head direction can influence the perception of gaze. In these two pictures, the face on the right seems to be gazing directly at the viewer whereas the face on the left appears to be looking slightly to the viewer's right. However, by covering the lower portions of each face, you can satisfy yourself that the eye regions of both are, in fact, identical.

More recently, a number of authors have made systematic investigations into this phenomenon. Gibson and Pick (1963) trained a "looker" to fix their gaze on each of seven horizontally arranged dots, separated from one another by an angle of $2.9^{\circ}$. The central point corresponded with the bridge of a subject's nose who was seated $2 \mathrm{~m}$ from the looker, whilst the remaining points extended to the left and right of the subject's head. The gaze fixations were made with the looker's head oriented directly toward the subject, or at an angle of $30^{\circ}$ to their left or $30^{\circ}$ to their right. The subjects' task was simply to judge whether or not the eyes were looking directly at them. Gibson and Pick established that when the looker's head was turned to one side, subjects tended to mistakenly judge gaze directed $2.9^{\circ}$ to the opposite side as actually 
looking directly at them. They concluded that the perception of eye-pointing is evidently influenced by the perception of head pointing.

Cline (1967) refined Gibson and Pick's (1963) conclusion, claiming that head orientation and eye-gaze direction interact so that the direction of attention is perceived as falling somewhere between these two positions. Rather than judge "mutual gaze", Cline's (1967, Experiment 2) subjects were asked to indicate the line of regard of a looker whose head was turned $30^{\circ}$ to the right whilst their eyes were directed at angles of either $0^{\circ}$ (i.e., directly at the subject), $4^{\circ}$ or $10^{\circ}$ to the left or right of the subject. Under these conditions, subjects' gaze judgements were found to err in the direction of the orientation of the looker's head. So for instance, when the looker's gaze was oriented directly towards the subject at the $0^{\circ}$ central point, judgements of the line of regard were actually made, on average, $3 \mathrm{~cm}$ to the right of this point.

The findings of Maruyama and Endo (1983) also support the hypothesis that the perception of another's attention represents an influence of both head and gaze orientations. They showed that subjects' perception of the direction of gaze in schematic faces was intermediate between the eyes' proper line of gaze and the orientation of the face. Like Cline (1967), Maruyama and Endo's findings indicated that the perceived line of gaze was, in fact, "towed" toward the orientation of the head. However, their data also suggested that the reverse was not the case. In other words, the processing of gaze direction was influenced by head orientation, but processing of head orientation was not influenced by the perception of the eyes' line of regard, a finding which is not predicted by the operation of Perrett et al's putative Direction of Attention Detector.

Finally, although still clearly supporting the view that the perception of gaze direction is influenced by the orientation of the head, Anstis, Mayhew and Morley (1969, Experiment 1) reported that the perceived direction of a looker's gaze in their experiment erred in the opposite direction to the turn of their head. In other words, if another individual was gazing into your eyes but their head was pointing toward your right shoulder, according to Anstis et al. (1969), you would perceive them as gazing somewhere near your left ear. 
Regardless of whether perceived gaze is shifted toward or away from the line of regard of the head, these studies suggest that the analysis of another individual's direction of attention involves a combination of information extracted from the eyes, and information extracted from the orientation of the head. Moreover, they hint that this interaction is more complex than a simple inhibition of information from a subordinate signal as Perrett et al. (1992) suggest. In the present work, the intention was to investigate further the operation of the putative DAD. More specifically, the aim was first to test the hypothesis that eye gaze and head orientation interact in the computation of social attention direction, and second to examine Perrett et al's hypotheses regarding the directionality of the interaction between these sources of information.

\section{Interference and Dimensional Interaction}

One way of exploring the interaction between different dimensions is to place them into conflict as in the McGurk effect (McGurk \& MacDonald, 1976), or the Stroop-type interference paradigm (Stroop, 1935; for a review see MacLeod, 1991). In the original version of the Stroop colour-word task, participants were slower to name the colour of the ink in which an incongruent colour word was printed (e.g., RED in blue ink) relative to a control condition of naming coloured squares. However, reading the colour word was largely unaffected by the ink colour in which it was printed. A similar asymmetry has been observed when printed words are combined with line drawings of familiar objects (e.g., Glaser \& Düngelhoff, 1984). Moreover, both pictureword and colour-word interference effects have been observed when the opposing dimensions are presented cross-modally (Cowan \& Barron, 1987; Schriefers \& Meyer, 1990; Shimada, 1990). More recently, Langton, O’Malley and Bruce (1996) applied the interference paradigm to the study of the interaction of gesture and speech in comprehension. They obtained symmetrical cross-modal interference effects when pointing gestures were paired with spoken directional words. That is, participants' reaction times (RTs) to the word "up" were slowed if they simultaneously saw a person pointing downwards compared with upwards. Similarly, when asked to respond to an upward pointing gesture, RTs were slower when participants heard a voice saying "down" compared with "up". 
These studies indicate how selective attention to a target stimulus on one dimension (e.g., gesture) can falter when that stimulus is paired with an attribute from some other dimension (e.g., spoken words). This breakdown in selective attention can be thought of as arising through an interaction, or "crosstalk" of information between processing channels at some level of information processing (e.g., Melara \& Marks, 1990a, 1990b). For example, Melara and Marks (1990a) used the similar Garner interference paradigm to establish symmetrical congruity effects in the classification of dimensions such as visual words and pitch, visual words and position, spoken words and position and spoken words and pitch. They concluded that these effects were caused by a bi-directional crosstalk of semantic information between the processing channels responsible for the analysis of the dimensions in question. So for instance, semantic information extracted from the spoken words "up" or "down" was considered to influence the extraction of meaning from a high or a low pitched tone and vice-versa. Similarly, Langton et al. (1996) attributed their symmetrical gesture-word interference effects to a bi-directional interaction between systems responsible for verbal and gestural processing. This crosstalk or integration of information was considered to occur at a central stage of processing, between perceptual encoding and response determination.

The interference paradigm therefore serves as a useful tool for gauging whether the stimuli under investigation are likely to come from so-called interacting dimensions (Melara \& Marks, 1990a) and, as a result, seems appropriate in the study of the analysis of another's social attention direction. If, as has been suggested, this computation involves the combination of information extracted from eye-gaze direction, head orientation and body posture, then a range of interference effects ought to be observed when attributes from these dimensions are placed into conflict. Moreover, the directionality of the interference effects (i.e., whether they are symmetric or asymmetric) will be informative regarding Perrett et al's (1992) hierarchy of influence of eye gaze, head and body orientation in the computation of social attention direction. Thus, in the experiments described below, a Stroop-Type interference paradigm was exploited in order to investigate any interactions between these social cues to attention direction. Experiments 1 and 2 used an intra-modal Stroop-Type paradigm to study the relationship between head 
orientation and eye-gaze direction. Experiment 3 used a cross-modal version of the task in order to explore further the relationship between head and gaze cues in the absence of selective attention to either of these signals.

\section{Experiment 1}

In the first experiment, participants were presented with photographic images of an individual whose head was oriented either upwards, downwards, to the left or to the right. However, in these images the individual's gaze was directed along either a congruent or incongruent line of regard to his head. Participants were asked to make a speeded keypress response to each of these social attention signals in separate blocks of trials. Perrett et al's (1992) direction of attention detector combines information from these signals in such a way that eyegaze direction will always inhibit head orientation, but not vice-versa. Therefore, under this hypothesis, eye gaze and head orientation are expected to produce an asymmetric pattern of interference. That is, the processing of head orientation will be influenced by the direction of the accompanying gaze, but classification of eye-gaze direction will be unaffected by the orientation of the head. If, on the other hand, head orientation plays more of a role in the determination of social attention direction than Perrett et al. suggest, a more symmetrical pattern of effects might be anticipated. Thus, RTs to head orientation will again be slowed by incongruent relative to congruent gaze directions, but in this case, RTs to gaze direction will also be influenced by tobe-ignored head orientations.

\section{Method}

Participants. These were 32 Open University Students who were taking part in a residential summer school at the University of Stirling.

Apparatus and Materials. An Apple Macintosh Performa 6200 controlled the stimulus displays and recorded responses. The visual stimuli consisted of eight digitised images of a male face (see Figure 2) which were displayed on an Apple Macintosh 14 inch colour monitor. These images were $8 \mathrm{~cm}$ in height and $5 \mathrm{~cm}$ in width and were viewed by participants seated 
approximately $0.7 \mathrm{~m}$ from the monitor. In each of these images the head was oriented approximately $16^{\circ}$ upwards or downwards, and approximately $30^{\circ}$ to the left or to the right of centre. In half of the images, the eyes were oriented in the same direction as the head (congruent stimuli). More specifically, for those stimuli oriented on the left/right axis, the eyes were oriented at $0^{\circ}$ with respect to the head, but for those oriented either upwards or downwards, the eyes were gazing at an angle of approximately $19^{\circ}$ with respect to the head. In other words, the gaze was directed in exactly the same direction as the head orientation in left-right heads, but was more extreme than the head direction in up-down oriented heads. In the other half of the images the eyes were gazing in the opposite direction to that of the head (incongruent stimuli). In left/right stimuli, the eyes were gazing approximately $72^{\circ}$ in the opposite direction to the line of regard of the head. This angle was approximately $51^{\circ}$ for the up/down stimuli.
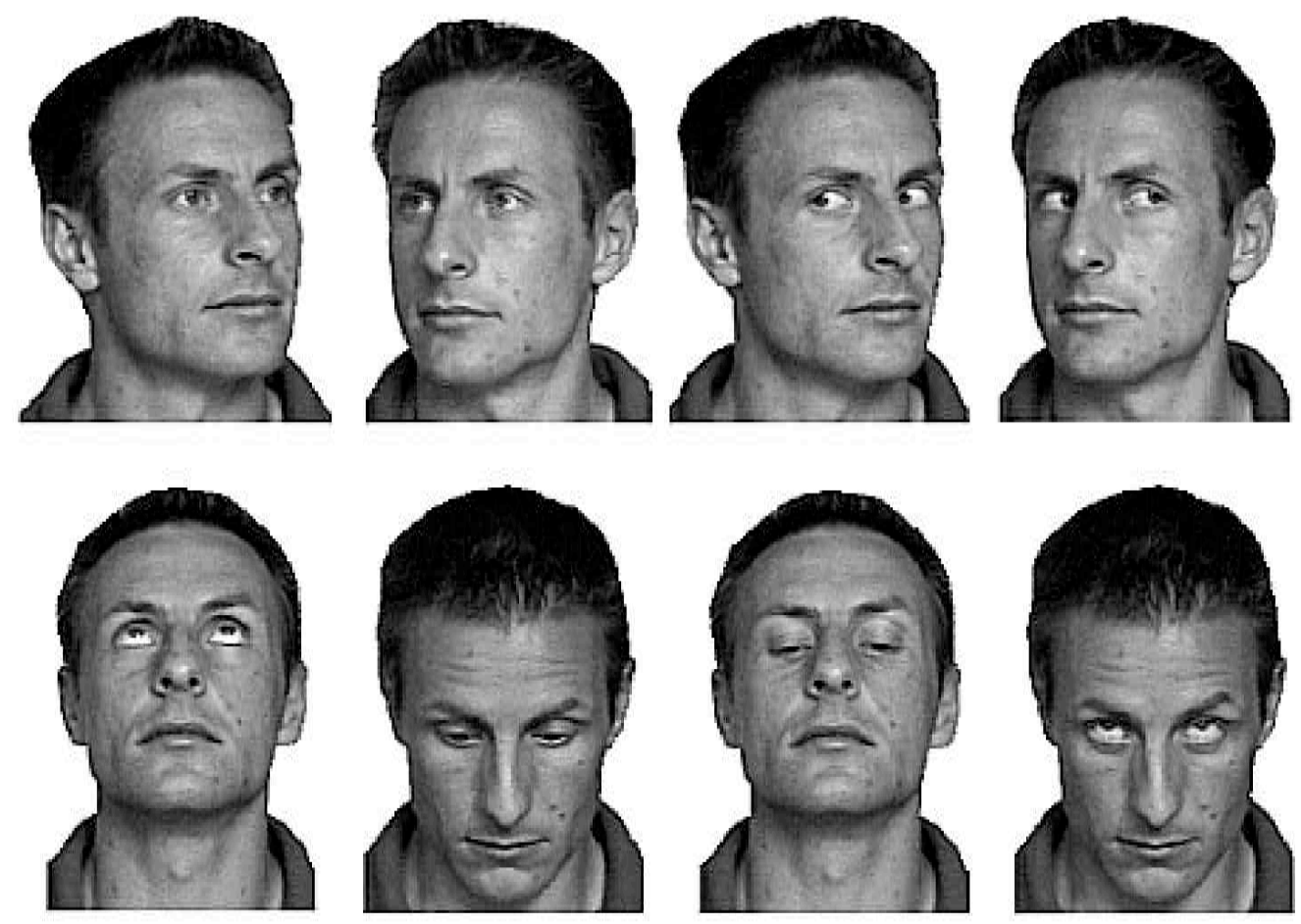

Figure 2. Reproductions of the digitised face stimuli used in Experiment 1.

Design. The materials were tested in a within subjects design with three factors: Response dimension (head or gaze), Congruity (congruent or incongruent) and Orientation (up/down or left/right). These factors were combined to yield eight equiprobable conditions. 
Procedure. On each trial, participants were asked to respond as quickly and accurately as possible to either the gaze or head direction of the visual stimulus which appeared in the centre of the screen. Participants executed their responses by depressing one of four buttons on the keypad area of the keyboard. They were asked to use their preferred hand to depress the keys, using their first and third fingers for the left-right decisions (keys 4 and 6 respectively) and their second finger for the up-down decisions (keys 8 and 5 respectively). Depresssion of any key terminated the presentation of the visual stimulus and stopped the timer. Following the response, the screen remained blank for 1000 ms before the start of the next trial.

Participants completed 160 experimental trials, comprising 20 trials in each of the eight cells of the experimental design. These trials were blocked by the response factor, and were presented in a random order within each block. Thus, in one block of trials participants responded to the orientation of the head and were asked to ignore the gaze, and in a second block of trials they responded to the gaze direction and were asked to ignore the orientation of the head. The order in which these blocks were presented was alternated between successive participants. A set of 16 practice trials were presented before each block, comprising two repetitions of each of the eight experimental stimuli. Both reaction times (RTs) and the proportion of errors were recorded as dependent variables in this experiment.

\section{Results}

In this and all other experiments reported in this article, outliers were removed from individual participants' scores by first removing those reaction times longer than 2, $000 \mathrm{~ms}$ and then those RT's greater than two standard deviations from each cell mean. The interparticipant mean correct RT's and standard deviations are summarised in Table 1. An inspection of this table reveals that, with the exception of responses to left-right oriented gaze, participants were slower and less accurate in incongruent compared with congruent conditions of the experiment. 
Table 1.

Mean Reaction Times (RTs; in milliseconds) and Percentage of Errors for Each Condition of Experiment 1.

\begin{tabular}{|c|c|c|c|c|c|c|}
\hline \multirow[b]{2}{*}{ Congruity } & \multicolumn{2}{|c|}{ Up/Down } & \multicolumn{2}{|c|}{ Left/Right } & \multicolumn{2}{|c|}{ Overall $M$} \\
\hline & RT & $\%$ of Errors & RT & $\%$ of Errors & RT & $\%$ of Errors \\
\hline & \multicolumn{6}{|c|}{ Response to Gaze } \\
\hline Congruent & 718 & .31 & 713 & 1.56 & 716 & .94 \\
\hline Incongruent & 776 & .78 & 673 & .47 & 725 & .63 \\
\hline \multirow[t]{2}{*}{$M$} & 747 & .55 & 693 & 1.02 & 720 & .79 \\
\hline & \multicolumn{6}{|c|}{ Response to Head } \\
\hline Congruent & 724 & .63 & 650 & 0 & 687 & .32 \\
\hline Incongruent & 846 & 6.41 & 712 & .47 & 779 & 3.44 \\
\hline$M$ & 785 & 3.52 & 681 & .24 & 733 & 1.88 \\
\hline
\end{tabular}

A 2 (response dimension) x 2 (congruity) x 2 (orientation) analysis of variance (ANOVA) conducted on participants' mean RT's supported the above observations. There were main effects of congruity $(\mathrm{F}(1,15)=38.31, \mathrm{p}<0.001)$ and orientation $(\mathrm{F}(1,15)=27.93, \mathrm{p}<0.001)$ as well as significant interactions between response and congruity $(F(1,15)=19.76, p<0.001)$, response and orientation $(\mathrm{F}(1,15)=13.15, \mathrm{p}<0.01)$ and between congruity and orientation $(\mathrm{F}(1,15)=65.30$, $\mathrm{p}<0.001)$. However, there was also a significant three-way interaction between response dimension, congruity and orientation $(F(1,15)=4.89, \mathrm{p}<0.05)$. Investigation of this interaction confirmed that participants' RTs were faster to congruent than to incongruent stimuli when responding to heads of both up-down and left-right orientations, and when responding to updown oriented gaze (p's $<0.001$ ). However, the opposite effect was found for responses to leftright oriented gaze. Here, RTs were faster to incongruent than to congruent stimuli $(\mathrm{p}<0.01)^{1}$. 
An inspection of Table 1 indicates that the error scores generally mirror the RT data. In fact the correlation between RTs and percentage of errors was 0.83 offering no evidence of a tradeoff between speed and accuracy. Because of the low rate of errors (the overall mean percentage error was only $1.33 \%$ ), coupled with the fact that performance in one cell of the design was flawless, no further analysis was conducted on these data.

\section{Discussion}

The results of this experiment clearly demonstrated that participants' responses to the orientation of another individual's head were severely influenced by the direction in which this individual was gazing. Reaction times to the direction in which the head was oriented were significantly slower when the eyes were gazing in the opposite direction to the head, compared with conditions in which the eyes and head were oriented in the same direction. A similar congruity effect was noted when participants were asked to respond to up/down gaze stimuli, suggesting that, contrary to the predictions derived from Perrett et al's DAD, head orientation is indeed able to influence the processing of eye gaze direction. These findings suggest that head and gaze direction are more mutually influential in the computation of social attention and clearly indicate that information derived from head orientation is not completely inhibited when the eyes are visible.

However, these conclusions are weakened by the observation of a reverse congruity effect for responses to horizontally displaced gazes. Here participants were faster to respond to the direction of gaze when the head was oriented in the opposite direction to the gaze's line of regard than when gaze and head orientation were in agreement. It seems as though participants found the task of determining the gaze direction for images in the latter condition to be rather harder than judging the gaze of left-right incongruent images.

One reason for this curious result may be that the direction of gaze in the incongruent heads is more discriminable than in the congruent heads. Baron-Cohen (1994) argues that the mechanism responsible for detecting gaze direction is sensitive to the contrast between the 
darker pupil/iris and the white sclera region of the eye. In support of this, Anstis et al. (1969) found that the apparent horizontal direction of gaze of an artificial eye could be altered simply by exposing different amounts of sclera on either side of the pupil. Gaze direction therefore seems to be determined by coding the position of the iris/pupil region relative to the sclera. The stimuli used in Experiment 1 were designed so that the gaze in corresponding congruent and incongruent images was directed toward the same point in external space. The result of this was that more of the sclera is visible in the left-right incongruent stimuli than in the equivalent congruent images. Accordingly, the former stimuli may have yielded a less ambiguous gaze direction code, and therefore faster classification RTs, than stimuli in the latter congruent condition. Thus, it may be more a priori appropriate to control for the amount of visible sclera in the preparation of the congruent and incongruent test stimuli, rather than ensuring that gazes in these images are directed toward the same location in space.

However, one problem with this account is that, as with the left/right stimuli, the amount of visible sclera was not equated across the vertically oriented head/gaze images. Despite this, participants did not seem to experience any associated problems in responding to these items. One reason for this may be that the simple presence or absence of visible sclera serves as a strong, unambiguous cue to vertically displaced gaze. In the downward directed gaze stimuli the sclera and most of the eye is occluded by the lid, whereas sclera is clearly visible below the iris when the gaze is oriented upwards (see Figure 2). Thus, the presence of sclera indicates an upward gaze, whilst its absence signals a downward gaze. So despite failing to control for the amount of visible sclera, the gaze directions in these stimuli may nevertheless have been equally discriminable. In this case, the congruity effects obtained when participants responded to vertically displaced gazes actually reflect the influence of information automatically encoded from the orientation of the head, rather than problems disambiguating gaze direction from any of the stimuli. persuasion

An alternative account of the reverse congruity effect is that the difficulties experienced with the left/right congruent stimuli arose because, in general, participants tend to judge the direction 
of seen gaze with respect to a frame of reference provided by the orientation of the head (e.g., Maruyama \& Endo, 1983). In all but the left-right congruent stimuli, the eyes are gazing upwards, downwards, to the left, or to the right relative to the line of regard of the head (see Figure 1). A coding scheme for gaze direction based on the orientation of the head would therefore yield a representation which would be consistent with the required response for that stimuli. However, an inspection of Figure 1 reveals that, for the congruent left-right stimuli, the eyes and head are oriented in exactly the same direction. In this case the coding system would yield a representation specifying that the gaze is directed straight ahead when the required response is actually either "left" or "right". Some additional processing would therefore be required to map this "straight ahead" gaze representation onto a representation specifying the direction of gaze with respect to the viewer, and thence onto the appropriate left or right response code.

A final suggestion is that the poor performance in making left-right gaze judgements to the congruent images is a result of the influence of head orientation on the perception of gaze direction (e.g., Anstis et al., 1969; Cline, 1967; Gibson \& Pick, 1963; Maruyama and Endo; 1983). In particular, Anstis et al. (1967) suggested that judgements of gaze err in the opposite direction to the turn of the head. In this case, a $30^{\circ}$ leftward gaze in the context of a head also turned $30^{\circ}$ toward the left would actually be perceived as oriented at a slightly less eccentric angle, directed more towards the observer. On the other hand, a gaze in the opposite direction to the orientation of the head (the incongruent images) might appear to be more eccentric than is actually the case. This kind of perceptual interaction may then have the effect of increasing the discriminablity of the incongruent, relative to the congruent gazes. This, in turn, would speed judgements of incongruent images, and slow those of congruent stimuli resulting in the reverse congruity effect.

However, whilst some kind of influence of head orientation on the perception of gaze direction may well be operating in this experiment, it seems unlikely to be sufficient to account for the interference effects for several reasons. First, head orientation only seems to produce a 
constant error in gaze perception of the order of $3^{\circ}$ which would seem to be too small to produce a significant influence on left/right gaze categorisations of the stimuli in the top row of Figure 2. Second, one might expect the same perceptual effects to be operating in the up/down stimuli, influencing the categorisations of congruent and incongruent stimuli in the same way. The findings of Experiment 1 indicate that this was not the case. Finally, the large discrepancy between the head and gaze cues in the incongruent conditions would seem to mitigate any perceptual effects. Indeed, Cline (1967) noted no perceptual interaction between head and gaze cues with a somewhat smaller discrepancy between the cues than was the case in the present experiment.

To summarise, the reversal of the congruity effect for left-right gaze judgements in Experiment 1 may be based on either the discriminability of the gaze direction per se, or the ambiguous nature of the gaze direction when coded relative to a frame of reference provided by the head. In Experiment 2, the ambiguity of the gaze direction in the left/right congruent images was reduced so that the gaze in these images would be at least as discriminable as that in the incongruent stimuli. Once again the individual's head was oriented either to the left, to the right, upwards or downwards, however in all these new stimuli his gaze was oriented left, right, up or down with respect to the angle of the head. This manipulation also had the effect of roughly equating the amount of visible sclera in the congruent and incongruent left/right images, which as suggested earlier, may be the more a priori appropriate way to motivate the design of these stimuli.

\section{Experiment 2}

\section{Method}

Participants. These were sixteen Open University students, none of whom had participated in Experiment 1. All had normal or corrected-to-normal vision.

Materials and Apparatus. A new set of stimuli were created which were broadly similar to those used in Experiment 1 (see Figure 3). The same gaze and head angles were used as in the 
previous experiment with the exception of the congruent left-right images. Here again the head was oriented at approximately $30^{\circ}$ to the left or right but with these new stimuli the gaze was oriented a further $16^{\circ}$ in the same direction as the head. In other respects, the materials and apparatus were identical to those used in Experiment 1.

Design and Procedure. These were identical to those used in Experiment 1.
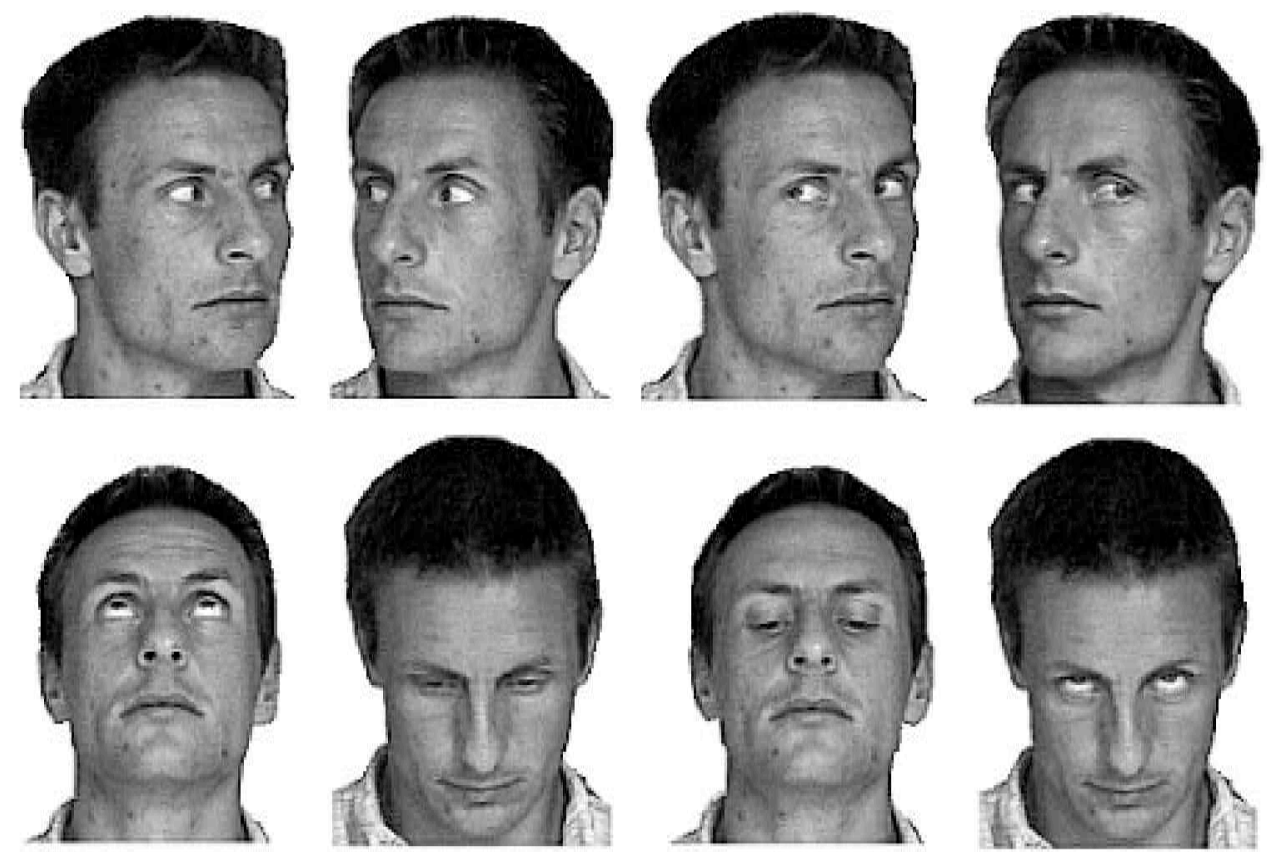

Figure 3. Reproductions of the digitised face stimuli used in Experiments 2 and 3.

\section{Results}

Once again, outliers were removed from individual subject's scores by first removing those reaction times longer than 2,000 $\mathrm{ms}$ and then those RT's greater than two standard deviations from each cell mean. The resulting mean correct reaction time scores and percentage of errors recorded in each condition are reported in Table 2. There are clear effects of congruity for responses to both gaze and head dimensions across both stimulus orientations. Overall, participants' RTs to congruent trials were $81 \mathrm{~ms}$ faster than their responses to incongruent trials. Responses to left-right oriented stimuli also seem to be generally faster than responses to updown stimuli. 
Table 2.

Mean Reaction Times (RTs; in milliseconds) and Percentage of Errors for Each Condition of Experiment 2.

\begin{tabular}{|c|c|c|c|c|c|c|}
\hline \multirow[b]{2}{*}{ Congruity } & \multicolumn{2}{|c|}{ Up/Down } & \multicolumn{2}{|c|}{ Left/Right } & \multicolumn{2}{|c|}{ Overall $M$} \\
\hline & RT & $\%$ of Errors & RT & $\%$ of Errors & RT & $\%$ of Errors \\
\hline & \multicolumn{6}{|c|}{ Response to Gaze } \\
\hline Congruent & 673 & .63 & 598 & .31 & 636 & .47 \\
\hline Incongruent & 763 & 2.81 & 651 & 1.25 & 707 & 2.03 \\
\hline \multirow[t]{2}{*}{$M$} & 718 & 1.72 & 625 & .78 & 672 & 1.25 \\
\hline & \multicolumn{6}{|c|}{ Response to Head } \\
\hline Congruent & 694 & 1.25 & 585 & 0 & 640 & .63 \\
\hline Incongruent & 810 & 1.88 & 651 & .63 & 731 & 1.26 \\
\hline$M$ & 752 & 1.57 & 618 & .32 & 685 & .95 \\
\hline
\end{tabular}

These observations were confirmed by a 2 (response dimension) x 2 (congruity) x 2 (orientation) ANOVA conducted on the RT data. This analysis revealed main effects of both congruity $(\mathrm{F}(1,15)=46.18, \mathrm{p}<0.001)$ and orientation $(\mathrm{F}(1,15)=38.45, \mathrm{p}<0.001)$. However, there was also a significant interaction between these factors $(F(1,15)=9.59, \mathrm{p}<0.05)$ which seems to arise because the effect of congruity was stronger for up-down oriented stimuli than for left-right images (103 ms versus $60 \mathrm{~ms}$ respectively). Simple main effects analysis confirmed that the effects of congruity were significant at both stimulus orientations, and also that RTs were superior for left-right compared with up-down stimuli for both congruent and incongruent trials (p's $<0.001$ ). The effect of orientation was further qualified by a significant interaction with the response dimension $(\mathrm{F}(1,15)=8.81, \mathrm{p}<0.05)$. The $\mathrm{RT}$ advantage for left-right over up-down stimuli was stronger for responses to the head direction than to the gaze direction (134 ms and 94 ms respectively; p's $<0.001)$. No other effects reached significance (p's $>0.1$ ) 
In Table 2 the error scores generally mirror the RT data; the overall mean error score was only $1.09 \%$. The correlation between overall mean RTs and mean error rates was 0.87 suggesting no evidence of a trade-off between speed and accuracy which might compromise interpretation of the RT data. Because of the low rate of errors, no further analysis was conducted on these data.

\section{Discussion}

In this experiment substantial congruity effects were obtained for both left-right and up-down stimulus orientations and for responses to both gaze and head direction. Moreover, these congruity effects were symmetrical in nature; responses to head orientation were influenced by the to-be-ignored gaze dimension to the same extent as classification of eye-gaze direction was influenced by the irrelevant orientation of the head. These findings corroborate those of Experiment 1 in confirming that head and gaze are both influential in social attention detection, and further suggest that information derived from the orientation of the head is not completely inhibited when processing eye-gaze direction as Perrett et al's (1992) model predicts.

However, it may be premature to use the relative magnitudes of the respective interference effects as evidence for or against a hierarchy of influence of gaze and head cues. The observation of a symmetrical pattern of interference suggests that either member of a pair of dimensions has a similar ability to disrupt selective attention to the other. However, it does not necessarily follow that a symmetry of interference between head and gaze entails that these cues enjoy an equal status in the analysis of social attention. It could be argued that the cognitive system would not normally be forced to select one attentional cue for further processing and response, whilst attempting to ignore or suppress information from the others. Under more normal circumstances, the direction of attention detector may well operate more passively, producing an output based on some kind of combination or integration of information from the various social attention signals. Moreover, it may be that the system endows one source of information, say gaze direction, with some kind of privileged status so that it carries more "weight" in the interaction process. So, although head direction may well interfere with the processing of gaze direction, the 
privileged gaze cues could nevertheless override head cues in a task which did not require selective attention to be directed toward the head channel. Thus, a more thorough investigation of the idea of a hierarchy of influence demands a task in which attention cues can be placed into conflict but where participants are not actually required to select any of these cues. Just such a task is employed in Experiment 3.

\section{Experiment 3}

Previous research has suggested that information derived from certain cues to the direction of social attention are able to influence the speeded classification of spoken words (see Langton, 1996; Langton. O’Malley \& Bruce, 1996). For instance, Langton et al. (1996) showed that participants' responses to spoken directional words were influenced by the simultaneous presentation of static "gestures" comprising head, eye and pointing cues to direction. Langton (1996) also reported data from a pilot experiment which indicated that a combination of head and gaze cues produced similar interference effects when participants were asked to respond to spoken words. One possibility is that these findings represent the influence of head and gaze together, or the output of the direction of attention detector, on the processing of spoken words. The important point is that by asking subjects to make their responses to the verbal stimulus, they are not required to select any of the visual information for further processing. Therefore, by manipulating the relationship between the two visual cues (e.g., head and gaze), and observing the influence that this manipulation has on subjects' responses to spoken words, we can observe the operation of the direction of attention detector in the absence of any selection process.

Thus, in Experiment 3 participants were asked to make a keypress response contingent on the identity of a spoken directional word (up, down, left, or right). Each word was accompanied by one of the head/gaze images used in Experiment 2. Therefore, the gaze direction was always either congruent or incongruent with the spoken word. Moreover, in half of the images, the head was in agreement with the direction of gaze, and in the remainder, the head disagreed with the gaze direction. If information from both gaze and head orientation are influential in processing attention direction, then we would expect the interference effect exerted by eye gaze direction on 
spoken words to be reduced, eliminated, or reversed when the head disagrees with the eyes. More specifically, if eye gaze is more important than head direction then the interference effect will be reduced, if gaze and head have a more equal status, the effect will be eliminated, and if head direction overrides eye gaze then the effect will be reversed.

\section{Method}

Participants. These were sixteen Open University students, none of whom had participated in Experiments 1-3. All had normal or corrected-to-normal vision.

Materials and Apparatus. The visual stimuli were the same eight images used in Experiment 2. In addition, the visual materials also contained a large question mark which subtended approximately $3^{\circ}$ of vertical visual angle. The verbal stimuli were recorded using Hypercard audio software and edited using "SoundEdit" software on a Macintosh IIci. Four spoken words ("up", "down", "left" and "right") were recorded and edited to be approximately the same length (0.8 secs). Each word was paired with each of the four visual images oriented on that axis to yield sixteen stimulus pairs which were presented as before using the SuperLab software on a Mactintosh Performa 6200.

Design. The audio-visual stimuli were presented in a within-subjects design with three factors: Gaze Congruity (congruent or incongruent) was determined by the relationship between the gaze direction and the spoken word, Head Agreement (agree or disagree), determined by the relationship between the orientation of the head and eye gaze direction, and Orientation (updown or left-right). Each of the sixteen test stimuli were presented ten times during the experiment yielding a total of 160 trials with 20 trials per condition.

Procedure. Each trial began with the simultaneous presentation of a visual head/gaze stimulus and a spoken word. Participants were asked to respond to the verbal information as quickly and accurately as possible using the same response keys as in Experiments 1 and 2. The onset of the auditory stimulus activated the timer which was stopped when the participant pressed any of the response keys. This response also terminated the presentation of the visual 
stimulus. The intertrial interval was set at $1000 \mathrm{~ms}$ following the execution of a response. Participants were also instructed to depress the space bar in response to a large question mark which occasionally appeared on the screen. Seven of these question marks appeared in each response block. These trials were included in order to ensure that the subjects actually watched the screen.

Partcipants completed a set of 18 practice trials on the task. These comprised two question mark trials and one of each of the 16 test stimuli. Following the practice block, participants were given two blocks of 95 trials (80 test test trials plus 15 question mark trials). Four practice trials were also presented immediately prior to the second experimental block. Trials were randomised within all blocks.

\section{Results}

The mean correct RTs and percentage of errors recorded in each condition are reported in Table 3. An inspection of this table reveals that when head and gaze direction agree with one another there is a clear $58 \mathrm{~ms}$ congruity effect which is reduced to $-1 \mathrm{~ms}$ when head and gaze disagree. Moreover, this pattern is maintained across both left-right and up-down stimulus orientations. 
Table 3.

Mean Reaction Times (RTs; in milliseconds) and Percentage of Errors for Each Condition of Experiment 3.

\begin{tabular}{|c|c|c|c|c|c|c|}
\hline \multirow[b]{2}{*}{$\begin{array}{c}\text { Gaze } \\
\text { Congruity }\end{array}$} & \multicolumn{2}{|c|}{ Up/Down } & \multicolumn{2}{|c|}{ Left/Right } & \multicolumn{2}{|c|}{ Overall $M$} \\
\hline & RT & $\%$ of Errors & RT & $\%$ of Errors & RT & $\%$ of Errors \\
\hline & \multicolumn{6}{|c|}{ Head Agree } \\
\hline Congruent & 682 & 0 & 656 & .94 & 668 & .47 \\
\hline Incongruent & 728 & 1.25 & 724 & 1.88 & 726 & 1.57 \\
\hline \multirow[t]{2}{*}{$M$} & 705 & .63 & 690 & 1.41 & 697 & 1.02 \\
\hline & \multicolumn{6}{|c|}{ Head Disagree } \\
\hline Congruent & 702 & .63 & 688 & .94 & 695 & .79 \\
\hline Incongruent & 698 & .31 & 689 & .31 & 694 & .31 \\
\hline$M$ & 700 & .47 & 689 & .63 & 695 & .55 \\
\hline
\end{tabular}

A 2 (Gaze Congruity) x 2 (Head Agreement) x 2 (Orientation) ANOVA conducted on the RT data supported these observations. This analysis revealed a main effect of gaze congruity $(\mathrm{F}(1,15)=24.52, \mathrm{p}<0.001)$ which was qualified by a significant interaction with head orientation $(F(1,15)=11.42, p<0.01)$. Simple main effects analysis confirmed that the effect of congruity was reliable when the head direction agreed with the gaze orientation $(\mathrm{p}<0.001)$ but failed to reach significance when head and gaze direction disagreed $(\mathrm{p}=0.89)$. This analysis also revealed significant effects of head agreement for both congruent and incongruent trials (p's $<0.05$ ). On gaze congruent trials, RTs were faster when the head agreed with the gaze direction than when it disagreed. However, this effect was reversed when the gaze direction and spoken word were incongruent. No other effects in the overall ANOVA approached significance (p's $>0.1)$.

The overall mean error score was $.78 \%$. The correlation between RTs and errors was 0.52 offering no evidence of a tradeoff between speed and accuracy. Because participants made 
relatively few errors, including an overall mean score of $0 \%$ in one of the cells of the design, no further analysis was conducted on these data.

\section{Discussion}

In Experiment 3 head and gaze cues were paired with spoken directional words and participants were asked to make a speeded response to the identity of the verbal stimulus. The gaze cues were either congruent or incongruent with the spoken word, and the head cues either agreed or disagreed with the direction of the eye gaze. As predicted, head and gaze cues which were in agreement with one another exerted large congruity effects on responses to spoken words. However, this congruity effect was completely eliminated when the head was oriented in the opposite direction to the eye-gaze cues. These findings clearly establish that information from both gaze and head orientation is extracted from the visual images even when participants are attempting to ignore these attention signals. Moreover, the results of Experiment 3, along with those of Experiments 1 and 2, indicate that head orientation plays a greater role in the processing of social attention direction than authors such as Baron-Cohen (1994) and Perrett et al. (1992) suggest. Clearly, information derived from the orientation of the head is not completely inhibited when the eyes are visible. On the contrary, the results of Experiment 3 suggest that, when placed in conflict, the information derived from the orientation of the head is sufficient to cancel out the information extracted from an incongruently directed gaze. Therefore, eye-gaze and head orientation are mutually influential in the computation of social attention direction.

\section{General Discussion}

Three experiments were conducted to investigate Perrett et al's (1992) ideas regarding the influence of head and gaze cues on the computation of another individual's direction of attention. In these experiments attributes from the dimensions under investigation were placed into conflict in a Stroop-type interference paradigm. According to Perrett et al's model, gaze cues should influence the processing of head cues, but the processing of gaze cues should be unaffected by 
any information extracted from head orientation. Consequently, an asymmetric pattern of interference effects was expected. However, Experiments 1 and 2 revealed symmetrical patterns of interference between head and gaze cues; to-be-ignored head cues produced as much interference on responses to gaze cues, as did to-be-ignored gaze cues on responses to head cues. Experiment 3 explored the same question using a cross-modal interference paradigm. In this study, head and gaze cues oriented in the same direction were found to interfere with responses to auditorily presented direction words. That is, to-be-ignored head and gaze cues produced slower RTs to incongruent than to congruent spoken words. However, this interference effect was eliminated when the gaze and head cues were oriented in opposite directions. This result indicates that, in the absence of selective attention to either cue, head and gaze signals are equally influential in the analysis of social attention direction. Taken together, these findings imply a larger role for head orientation in the analysis of social attention than Perrett and his colleagues have suggested.

What kind of model might account for this pattern of results? One possiblility is that they reflect the operation of some kind of social attention system containing channels processing various different social attention signals, including head and gaze direction (cf. Perrett et al., 1992). However, unlike Perrett et al's account, the links between these processing channels would be bi-directional allowing any one social attention signal to exert some kind of influence over the processing of any other. In this way, the output of the system represents a combined social attention signal, reflecting the influence of a number of different social cues. This kind of model could explain the mutual influence of head and gaze found in Experiments 1 and 2, as well as the cross-modal effects noted in Experiment 3. In the latter, it is the combined output of the social attention system which actually influences the processing of spoken words.

However, it is also possible that the findings of Experiment 3 represent the independent, rather than combined influences of head and gaze on the processing of spoken words. Experiment 3 showed that the congruent/incongruent relationship between gaze direction and the spoken word interacted with the agreement of the head with the direction of gaze. In this 
experiment the effect exerted by gaze direction on responses to spoken words was eliminated when the head was oriented in the opposite direction to the angle of gaze. However, by recoding the data it is possible to examine whether the congruity of the head with spoken words is additive or interactive with the congruity of the gaze cues with the same spoken words. Should gaze and head congruity interact, this would be good evidence for a combined influence of a head/gaze signal on the processing of spoken words. On the other hand, an additive pattern of effects would indicate independent influences of gaze and head cues.

In order to explore these alternatives, the head agreement factor in Experiment 3 was replaced with a Head Congruity factor. This variable referred to the congruent/incongruent relationship between the head orientation and the directional word, rather than the relationship between the head and gaze directions. The RTs for each participant were rescored accordingly, and the resultant means entered into $2 \times 2 \times 2$ ANOVA with gaze congruity, head congruity and orientation as within-subjects factors. This analysis yielded main effects of both gaze congruity $(\mathrm{F}(1,15)=24.52, \mathrm{p}<0.001)$ and head congruity $(\mathrm{F}(1,15)=11.42, \mathrm{p}<0.001)$, but critically, no interaction between these factors $(\mathrm{p}=0.69)$. Head orientation and gaze direction therefore exert independent effects on responses to spoken words. Thus, rather than reflecting the influence of a combined head/gaze signal on responses to spoken words, the results are more consistent with a model where head and gaze are analysed in parallel by separate systems, with the outputs of each exerting an effect on the processing of spoken directional words.

Langton, O'Malley and Bruce (1996) argued that the likely locus of the interference effect they noted between spoken directional words and pointing gestures was at a stage in processing after the independent identification of the component dimensions, but prior to the determination, programming and execution of a response. More recent work has suggested that the processing of these "gestures" actually comprised the parallel processing of head and/or gaze orientation and the actual pointing hand gesture itself (Langton \& Bruce, submitted). Meanwhile, the present findings suggest that head and gaze cues are also analysed by separate systems operating in parallel. Thus, the general idea is that a number of separate mechanisms exist which process 
signals such as head orientation, gaze direction, pointing gestures and spoken words. These systems feed their outputs into a processing stage or stages where the directional information encoded from the cues is somehow pooled before a response can be selected and executed. This means that, if present, any of these sources of information will be available to influence a directional decision, and interference effects will always emerge whenever copresent directional signals give rise to conflicting codes.

Massaro and his colleagues have developed a model of information integration which might well account for this "pooling" of information derived from head, gaze and spoken directional words. (e.g., Thompson \& Massaro, 1994; Massaro \& Egan, 1996; Massaro \& Friedman, 1990). Their so-called Fuzzy Logical Model of Perception (FLMP) has been applied to a number of domains including interactions between gesture and speech, and between facial emotional expressions and voice. In the FLMP, the two sources of information are evaluated independently and then integrated with a rule best described as a multiplicative algorithm. That is, the two signals are combined in such a way that the least ambiguous source of information carries more weight in the integration. This process yields a single overall description which is then matched against prototype descriptions in memory. Finally, an identification decision is made on the basis of the relative goodness of match of the integrated stimulus information with these stored prototype descriptions.

Can an account based on the FLMP explain the interactions between the social attention cues observed in the present experiments? One problem is that in the FLMP decisions are based on an integrated representation of information from the component dimensions. This means that following the integration process the integrity of the original signals will have been lost. Accordingly, one would expect participants to make a high proportion of errors when asked to base their responses on information from one of the original dimensions. Although the RT data from Experiments 1-3 suggest that participants' selective attention to the relevant stimulus faltered to some degree, the very small proportion of errors obtained in these studies indicates that participants were nonetheless successful in selecting information from the target dimension. 
Their decisions seem to be based on intact rather than integrated representations of the original dimensional information. Thus, it may be that the mutual influence of gaze and head orientation is better accounted for by some kind of non-integrative combination of information. For instance, it may be that the context established by one cue (e.g., gaze) can act to modulate or bias the decision process to a second cue (e.g., head angle) and vice-versa. In the cross modal case (Experiment 3), both head and gaze direction would act independently to modulate decisions to spoken words. Clearly, the precise nature of the combination or pooling process awaits further research.

\section{Conclusion}

The three experiments reported here demonstrate that head orientation and gaze direction interact mutually in the processing of another's direction of attention. Moreover, these studies indicate that, contrary to the hypotheses derived from the work of Perrett et al (1992), information derived from the orientation of the head is not completely inhibited when gaze direction is visible. Instead, it is suggested that under normal circumstances the orientations of head and gaze are computed in parallel before being combined at some later stage or stages of processing so that both cues are able to influence decisions concerning the direction of social attention. 


\section{References}

Anstis, S. M., Mayhew, J. W. \& Morley, T. (1969). The perception of where a face or television 'portrait' is looking. American Journal of Psychology, 82, 474-489.

Argyle, M., \& Cook, M. (1976). Gaze and mutual gaze. Cambridge, UK.: Cambridge University Press.

Baldwin, D. (1991). Infants' contribution to the achievement of joint reference. Child Development, 62, 875-890.

Baron-Cohen, S. (1994). How to build a baby that can read minds: Cognitive mechanisms in mindreading. Cahiers de Psychologie Cognitive, 13, 513-552.

Baron-Cohen, S. (1995a). The Eye Direction Detector (EDD) and the Shared Attention Mechanism (SAM): Two cases for evolutionary psychology. In C. Moore \& P. Dunham (Eds.), Joint attention: Its origins and role in development (pp. 41-59). Hillsdale, NJ: Lawrence Erlbaum Associates.

Baron-Cohen, S. (1995b). Mindblindness: An essay on autism and theory of mind. Cambridge, MA: MIT Press/Bradford Books.

Byrne, R., \& Whiten, A. (1991). Computation and mindreading in primate tactical deception. In A. White (Ed.), Natural Theories of Mind Oxford: Basil Blackwell.

Campbell, R., Heywood, C. A., Cowey, A., Regard, M., \& Landis, T. (1990). Sensitivity to eye gaze in prosopagnosic patients and monkeys with superior temporal sulcus ablation. Neuropsychologia, 28, 1123-1142.

Cline, M. G. (1967). The perception of where a person is looking. American Journal of Psychology, 80, 41-50. 
Cowan, N., \& Barron, A. (1987). Cross-Modal auditory visual Stroop interference and possible implications for speech memory. Perception and Psychophysics, 41, 393-401.

Driver, J., Davis, G., Kidd, P., Maxwell, E., Ricciardelli, P., \& Baron-Cohen, S. (in press). Shared attention and the social brain: Gaze perception triggers automatic visuospatial orienting in adults. Visual Cognition.

Gibson, J. J., \& Pick, A. (1963). Perception of another person's looking. American Journal of Psychology, 76, 86-94.

Glaser, W. R., \& Düngelhoff, F. J. (1984). The time course of picture-word interference. Journal of Experimental Psychology: Human Perception and Performance, 10, 640-654.

Heywood, C. A., \& Cowey, A. (1992). The role of the 'face cell' area in the discrimination and recognition of faces by monkeys. Philosophical Transactions of the Royal Society of London, Series B., 335, 31-38.

Jenkins, J. (1998) Detecting social signals from the face. Unpublished Doctoral Dissertation, University of Stirling, Stirling, UK.

Kendon, A. (1967). Some functions of gaze direction in social interaction. Acta Psychologica, $26,22-63$.

Langton, S. R. H. (1996) Interference between gestures and words. Unpublished Doctoral Dissertation, University of Nottingham, Nottingham, UK.

Langton, S. R. H., \& Bruce, V. (in press). Reflexive visual orienting in response to the social attention of others. Visual Cognition.

Langton, S. R. H., \& Bruce, V. (submitted). You must see the point: Automatic processing of cues to the direction of social attention. Journal of Experimental Psychology: Human Perception and Performance. 
Langton, S. R. H., O'Malley, C., \& Bruce, V. (1996). Actions speak louder than words: Symmetrical cross-modal interference effects in the processing of verbal and gestural information. Journal of Experimental Psychology: Human Perception and Performance, 22.

MacLeod, C. M. (1991). Half a century of research on the Stroop effect: An integrative review. Psychological Bulletin, 109, 163-203.

Maruyama, K., \& Endo, M. (1983). The effect of face orientation upon apparent direction of gaze. Tohoku Psychologica Folia, 42, 126-138.

Massaro, D. W., \& Egan, P. B. (1996). Perceiving affect from the face and voice. Psychonomic Bulletin and Review, 3, 215-221.

Massaro, D. W., \& Friedman, D. (1990). Models of integration given multiple sources of information. Psychological Review, 97, 225-252.

McGurk, H., \& MacDonald, J. (1976). Hearing lips and seeing voices. Nature, 264, 746-748.

Melara, R. D., \& Marks, L. E. (1990a). Processes underlying dimensional interactions: Correspondences between linguistic and nonlinguistic dimensions. Memory and Cognition, $18,477-495$.

Melara, R. D., \& Marks, L. E. (1990b). Perceptual primacy of dimensions: Support for a model of dimensional interaction. Journal of Experimental Psychology: Human Perception and Performance, 16, 398-414.

Mendelsohn, M., Haith, M., \& Goldman-Rakic, P. (1982). Face scanning and responsiveness to social cues in infant monkeys. Developmental Psychology, 18, 222-228.

Perrett, D. I., \& Emery, N. J. (1994). Understanding the intentions of others from visual signals: Neurophysiological evidence. Cahiers de Psychologie Cognitive, 13, 683-694. 
Perrett, D. I., Hietanen, J. K., Oram, M. W., \& Benson, P. J. (1992). Organisation and functions of cells responsive to faces in the temporal cortex. Philosophical Transactions of the Royal Society of London, Series B., 335, 23-30.

Perrett, D. I., Smith, P. A. J., Potter, D. D., Mistlin, A. J., Head, A. S., Milner, A. D., \& Jeeves, M. A. (1985). Visual cells in the temporal cortex sensitive to face view and gaze direction. Proceedings of the Royal Society of London, B223, 293-317.

Schriefers, H., \& Meyer, A. S. (1990). Experimental note: Cross-modal, visual-auditory pictureword interference. Bulletin of the Psychonomic Society, 28, 418-420.

Shimada, H. (1990). Effect of auditory presentation of words on colour naming: the intermodal Stroop effect. Perceptual and Motor Skills, 70, 1155-1161.

Stroop, J. R. (1935). Studies of interference in serial verbal reactions. Journal of Experimental Psychology, 18, 643-662.

Thompson, L. A., \& Massaro, D. W. (1994). Children's Integration of Speech and Gestures in Comprehension. Journal of Experimental Child Psychology, 57, 327-354.

Vecera, S. P., \& Johnson, M. H. (1995). Gaze detection and the cortical processing of faces: Evidence from infants and adults. Visual Cognition, 2, 59-87.

Von Grünau, M., \& Anston, C. (1995). The detection of gaze: A stare in the crown effect. Perception, 24, 1297-1313.

Wollaston, W. H. (1824). On the apparent direction of eyes in a portrait. Philosophical Transactions of the Royal Society, London, 247-256. Cited in Bruce, V., \& Young, A. (1998). In the eye of the Beholder: The science of face perception. Oxford: Oxford University Press. 


\begin{abstract}
Author Note
The author thanks Vicki Bruce for her helpful discussions on this topic, and an anonymous reviewer for comments on an earlier version of the manuscript. Correspondence concerning this article should be addressed to Stephen R. H. Langton, Department of Psychology, University of Stirling, Stirling, FK9 4LA, Scotland. Electronic mail may be sent to srhl1@stirling.ac.uk.
\end{abstract}




\section{Footnote}

${ }^{1}$ For ease and clarity of presentation we have chosen to present the analyses of the RT data from Experiments 1 and 2 collapsed over block order. Neither the pattern of effects, nor the conclusions drawn from these studies are at all influenced when block order is taken out as a between-subjects factor, or when participants' data from each of the two block orders are analysed separately. 